Advance Journal of Food Science and Technology 5(2): 127-131, 2013

DOI:10.19026/ajfst.5.3231

ISSN: 2042-4868; e-ISSN: 2042-4876

(C) 2013 Maxwell Scientific Publication Corp.

Submitted: August 31, $2012 \quad$ Accepted: October 19, 2012

Published: February 15, 2013

\title{
Research Article \\ Liquid Culture of Adventitious Roots is a Potential Alternative to Field Cultivation for Psammosilene tunicoides, a Rare and Endangered Endemic Medicinal Plant
}

\author{
${ }^{1,2}$ Zongshen Zhang, ${ }^{2}$ Zhenyan Yu, ${ }^{1}$ Zhaoxia Jin, ${ }^{1}$ Jun Liu and ${ }^{1}$ Yunfang Li \\ ${ }^{1}$ Department of Biological Engineering, Dalian Polytechnic University, Qinggongyuan, \\ Ganjingzi District, Dalian, 116034, P.R. China \\ ${ }^{2}$ Henan Province Health Albert Med-Tech Co., Ltd, Zhengzhou, 450001, P.R. China
}

\begin{abstract}
The aim of this study was to establish an adventitious roots culture system for sterile plantlet segments of P. tunicoides and improved the accumulation of total saponins in cultured roots. Psammosilene tunicoides is a native Chinese plant with high commercial value as medicinal herb. Combination of NAA and IBA significantly affected the adventitious roots formation on agar-solided $\mathrm{B}_{5}$ media and a maximal induction rate of $83 \%$ was obtained at $24 \pm 2^{\circ} \mathrm{C}$ with a photoperiod of $12 \mathrm{~h}$. With a shaking of $110 \mathrm{rpm}$ in darkness, transferring the detached adventitious roots to the growth regulator free $1 / 2 \mathrm{~B}_{5}$ liquid media notably increased the biomass production compared to that on solid media over a 30-day-culture period. Further analyses showed that more saponins could be accumulated in the liquid culture than in the solid culture and the addition of exogenous oxalic acid to the liquid media could enhance the accumulation of total saponins in adventitious roots. These results suggested that adventitious roots culture will be an efficient alternative to the field cultivation of intact plants for the production of useful natural compounds from $P$. tunicoides.
\end{abstract}

Keywords: Adventitious roots, liquid culture, Psammosilene tunicoides, saponin

\section{INTRODUCTION}

Psammosilene tunicoides W. C. Wu et C. Y. Wu is a herb species in the Psammosilene genus of Caryophyllaceae. Endemic to the South-Western region of China, this plant is primarily distributed at an elevation of 2600-3200 $\mathrm{m}$, in the crevices of rocks (Wang et al., 2002). Its root has been widely used as medicinal herbs in China for exhibiting various therapeutic effects, such as stopping bleeding, relieving pain and promoting blood circulation (Deng et al., 2009). Research results have identified the active components of Psammosilene as saponins and cyclic peptides. Crude saponins obtained from this plant have been shown to possess a strong activity of painrelieving and anti-inflammatory (Wang et al., 2006). Over the past decade, market demand for $P$. tunicoides has dramatically increased in China and other Asian countries and $P$. tunicoides has been facing an imminent danger of extinction due to the habitat destruction and illegal collection. In face of the acute scarcity of the plant, China has enlisted this plant as an endangered and rare plant species, inevitably affecting its market supply in future. Hence, it's urgent and necessary to seek new means to reproduce this plant. Adventitious roots induced by in vitro methods showed high rates of proliferation and active secondary metabolism and thus offers a viable alternative for providing $P$. tunicoides in the pharmaceutical industry. Adventitious roots are of natural product, grow vigorously in phytohormone-free media and possess great potential to accumulate valuable secondary metabolites. Cultivation of adventitious roots has been suggested as an alternative for natural compounds production. In the field of plant biotechnology, hairy roots, induced by $\mathrm{Ri}$ plasmid insertion, has been reported to be a promising pathway for producing rare and endangered medicinal plant species (Zhong et al., 2002). However, compared with hairy root cultures and field cultivation, this approach is safer, more stable and easier for management (Sudha and Seeni, 2001). To our knowledge, however, culturing adventitious roots of $\mathrm{P}$. tunicoides has not been reported yet. In this paper, we developed a liquid culture system for $P$. tunicoides adventitious roots and laid a foundation for large-scale production of secondary metabolites in $P$. tunicoides adventitious roots and could be expected to be an alternative to filed cultivation or wild resources.

\section{MATERIALS AND METHODS}

Plant material: Sterile plantlets of $P$. tunicoides were grown on agar-solidified $B_{5}$ medium supplemented with sucrose $25 \mathrm{~g} / \mathrm{L}+6$-BA $0.05 \mathrm{mg} / \mathrm{L}$ + IAA $0.05 \mathrm{mg} / \mathrm{L}$ at

\footnotetext{
Corresponding Author: Zongshen Zhang, Department of Biological Engineering, Dalian Polytechnic University, Qinggongyuan, Ganjingzi District, Dalian, 116034, P.R. China

This work is licensed under a Creative Commons Attribution 4.0 International License (URL: http://creativecommons.org/licenses/by/4.0/).
} 
Adv. J. Food Sci. Technol., 5(2): 127-131, 2013

a temperature of $22-24^{\circ} \mathrm{C}$ and illuminated by 1800 lux with a photoperiod of $12 \mathrm{~h}$ for 25 days.

Adventitious root cultures were induced from the sterile plantlets of $P$. tunicoides and sub cultured every 30 days by adding $0.1 \mathrm{~g}$ roots (about 10 pieces of roots) to a $100 \mathrm{~mL}$ flask containing $25 \mathrm{~mL}$ of $\mathrm{B}_{5}$ liquid medium without phytohormone. Cultures were shaken using a rotary shaker at $110 \mathrm{rpm}$ under $22 \pm 2^{\circ} \mathrm{C}$ in the dark.

Callus, from the sterile plantlets of $P$. tunicoides, was induced and maintained on the agar-solidified $\mathrm{B}_{5}$ (sucrose $25 \mathrm{~g} / \mathrm{L}+6$-BA $0.05 \mathrm{mg} / \mathrm{L}$ + IAA $0.05 \mathrm{mg} / \mathrm{L}$ ) under the same culturing conditions with that of sterile plantlets but without light. And the cultures were subcultured every 30 days.

Elicitor treatment: The experiment of in vivo elicitation with oxalic acid was carried out according to the procedure described by Zhang et al. (2009) with modifications [19]. Oxalic acid was dissolved in distilled water. The $\mathrm{pH}$ of this solution was adjusted to 5.6 with $\mathrm{NaOH}$ and autoclaved for 30 min which was added to the medium at specified time under sterile condition. The elicitor was added at a final concentration of $10^{-5} \mathrm{~mol} / \mathrm{L}$ in $0,7,15$ and 20-day-old suspension cultures, respectively and incubated for a further period until the $30^{\text {th }}$ day of culture. Control cultures were treated with sterile distilled water.

Extraction and estimation of total saponins: On the specified days post of culturing, the adventitious roots, together with callus and plantlets, were harvested and dried to a constant weight at $60^{\circ} \mathrm{C}$ in an electric oven and then ground into fine powder. Total saponins were extracted with $80 \%$ methanol and ultrasonicated for $1 \mathrm{~h}$ at $40^{\circ} \mathrm{C}$. The extract was centrifuged at $1000 \mathrm{rpm}$ for 10 min and evaporated to dryness. The dried pellet was reextracted with water-saturated $n$-BuOH. After evaporating the $n-\mathrm{BuOH}$ phase phase to dryness, a weighed pellet was dissolved in water to some volume.

Total saponins content was determined according to the procedure previously published with subtle modification (Tian et al., 2009; Zhang and Wang, 2009). Five $\mu \mathrm{L}$ of extraction solution was mixed with $0.5 \mathrm{~mL}$ vanillin solution (8\%, dissolved with ethanol) and $5 \mathrm{~mL} \mathrm{H}_{2} \mathrm{SO}_{4}(72 \%)$ and then the mixture was incubated at $60^{\circ} \mathrm{C}$ under water bath for $10 \mathrm{~min}$, after rapid cooling to room temperature, the absorbance was measured at $544 \mathrm{~nm}$. Calibration curve were established with ginsenoside $R e$ as reference saponin.

Statistic analysis of data: The growth ratio was calculated using the following equation and recorded as means \pm S.D. of data obtained from three experiments.
Growth ratio $=\left(W_{2}-W_{1}\right) / W_{1}$, where $W_{1}$ is the dry weight of biomass in time $t_{1}(\mathrm{~g})$ and $W_{2}$ represents the harvested dry weight of biomass $(g)$ in time $t_{2}$.

Relative growth rate was calculated from dry weight and meant the average productivity per day and was from the formula of $\left(W_{t}-W_{0}\right) /\left(W_{0} \cdot t\right), W_{0}$ is the dry weight of the inoculums $(g), W_{t}$ is the dry weight of biomass $(g)$ at time $t(\mathrm{~d})$.

\section{RESULTS AND DISCUSSION}

Induction and culture of adventitious roots from segments of sterilized plantlets: We first tested the effects of plant growth regulators on the formation of adventitious roots on solid $B_{5}$. $(2-3 \mathrm{~cm})$ segments of sterilized plantlets were used as explants for initiation of adventitious roots. At the $9^{\text {th }}-12^{\text {th }}$ day of inoculation in dark, adventitious roots formation was observed form the cut end. As shown in Table 1, the highest frequency of adventitious root induction occurred in the combination of $0.05 \mathrm{mg}$ IBA + $0.1 \mathrm{mg}$ NAA, with a maximum of $83 \%$, followed by combination of $0.1 \mathrm{mg}$ IBA + 0.1 mg NAA (39\%), 0.2 mg IBA + 0.1 mg NAA (9\%) and $0.4 \mathrm{mg}$ IBA + $0.1 \mathrm{mg}$ NAA (3\%). Different morphologies of the adventitious roots were also recorded between different treatments and they could be classified into three types:

- Type 1: Thin and short roots in the presence of IBA alone (Fig. $1 \mathrm{C}$ )

- Type 2: Fast growing and thick roots in the presence of NAA alone (Fig. $1 \mathrm{~B}$ )

- Type 3: Fast growing and highly branched roots in high ratio of NAA to IBA relatively (Fig. 1 D)

In our study, we found that auxins involves the root formation process, in agreement with previous results that application of exogenously applied auxins would aid adventitious rooting, although this may not be a universal requirement across species (Staden and Harty, 1988; Cristofori et al., 2010). Clearly, our data showed that the type and concentration of auxins strongly influenced the formation of adventitious roots (Table 1, Fig. 1) and also further indicated that IBA is an effective inducer of adventitious root formation. Low ratio of IBA to NAA appeared more efficient to induce adventitious rooting.

Establishment of liquid culture system for adventitious roots of $\boldsymbol{P}$. tunicoides: After induction and culturing on solid B5 for 14-17 days, the adventitious roots were detached from the explants aseptically and transferred into the liquid 1/2 B5 media

Table 1: Effects of IBA \& NAA on the formation of adventitious roots of $P$. tunicoides cuttings

\begin{tabular}{llll}
\hline Combinations of IBA \& NAA & Numbers of explants & Numbers of adventitious roots & Percentage of rooting (\%) \\
\hline 0.05 IBA + 0.1 NAA & 90 & $75 \pm 2.5$ & 83 \\
0.1 IBA + 0.1 NAA & 90 & $39 \pm 1.3$ & 43 \\
0.2 IBA + 0.1 NAA & 90 & $9 \pm 0.80$ & 10 \\
0.4 IBA + 0.1 NAA & 90 & $3 \pm 0.60$ & 3 \\
\hline
\end{tabular}




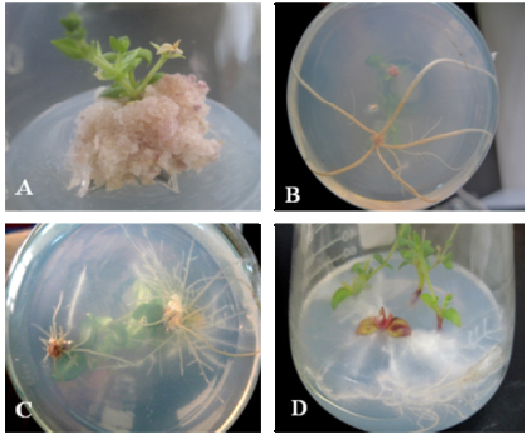

Fig. 1: The effects of IBA and NAA on the formation of adventitious roots at the segments base of $P$. tunicoides at 17 days (A) only formation of callus when the ratio of NAA/IBA was more than 4, (B) Less branch roots occurred at the main root in the presence of NAA alone, (C) Addition of IBA alone, thin and short roots in the presence of IBA alone, (D) In low ration of IBA to NAA, relatively highly branched roots compared with that treated with NAA in (B) and $(\mathrm{C})$

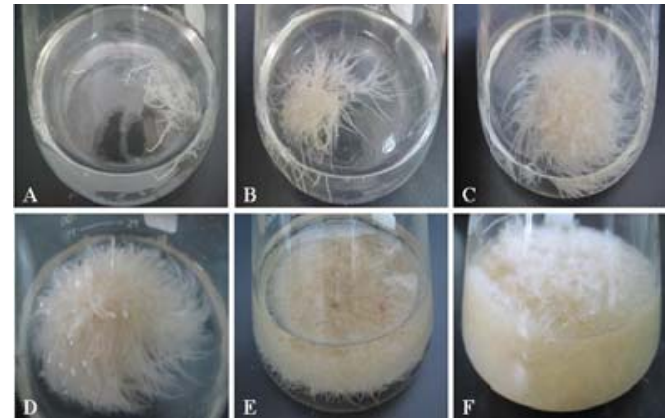

Fig. 2: Profiles of multiplication of adventitious roots in the liquid B5 showing high growth rate (A) Initiation stage, (B) 7 days, (C) 13 days, (D) 17 days, (E) 25 days, (F) 45 days

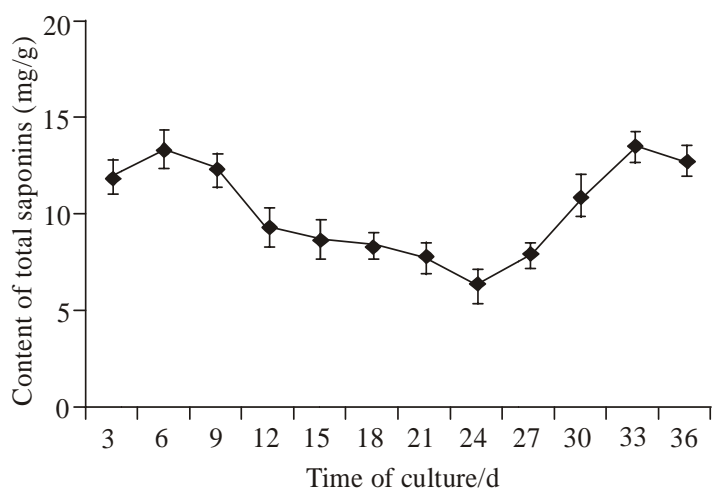

Fig. 3: Changes in total saponins concentrations during liquid culture of hairy roots with time

without phytohormone. When the initial inoculum was $0.624 \mathrm{~g} / 100 \mathrm{~mL}$, it reached a maximum of $8.250 \mathrm{~g} / 100$ $\mathrm{mL}(\mathrm{FW})$ at $30^{\text {th }}$ day (Table 2), 13.4 times higher than that of the initial inoculums. The volume of culture medium decreased to almost one third of the original volume by the time of collection, likely due to the intake of water and nutrients by growing adventitious roots.

With the prolonging of culture time, the adventitious roots in liquid media became tangled and formed a round shape of roots. The top and bottom regions of the adventitious root proliferated significantly and appeared yellowish white in color and the inside of the adventitious root differentiated into old and senescent tissues in brownish yellow (Fig. 2), consistent with previous observations for other plant species (Min et al., 2007). This could be due to the exposure of periphery tissues to sufficient nutrients and oxygen; by contrast, the inside of cultures was of restricted supply of nutrients and oxygen.

Correlation of accumulation of total saponin with culture time: Overall, the pattern of saponins accumulation in adventitious roots displayed a "V" trend. An increase in the amount of saponins was initially observed for the first 7 days of incubation (13.4 $\mathrm{mg} / \mathrm{g}$ dry mass at $7^{\text {th }}$ day). Beyond this period, the content of saponins showed a gradual decrease and arrived at the minimal value at 24 days and then followed by a rise in the amount of saponins accumulation up to 33 days of incubation (Fig. 3). Taken together, the data of both Table 2 and Fig. 3 demonstrated that there is a reciprocal correlation between the secondary metabolite accumulation and the growth period of adventitious roots. And these results further suggested that the biosynthesis of saponin in the adventitious roots of $P$. tunicoides was in a mode of cell differentiation dependence.

Comparison of total saponins between liquidcultured adventitious roots and other materials: The present study indicated that the adventitious roots of $P$. tunicoides resulted in a higher production of saponins in liquid culture $(16.33 \pm 0.85 \mathrm{mg} / \mathrm{g} \mathrm{DW})$ compared to callus $(11.71 \pm 0.90 \mathrm{mg} / \mathrm{g} \mathrm{DW})$ (Wei et al., 2010), with a comparable production of that for sterile seedlings $(17.85 \pm 1.25 \mathrm{mg} / \mathrm{g} \quad \mathrm{DW})$. Also, the adventitious roots got the highest relative growth rate among these different cultures or explants and achieved the best yield of total saponins per liter basis (Table 3). In addition, liquid culture of $P$. tunicoides brought about the excretion of some saponins into the media during liquid culture of adventitious roots of $P$. tunicoides. Taken together, these data suggest several advantages to use adventitious roots of $P$. tunicoides for the production of active compounds with medicinal value in the liquid system developed in this study.

Effects of a biotic elicitors on the accumulation of biomass and secondary metabolites: Although the possibility of enhancing accumulation of secondary products by the addition of biotic or abiotic elicitors to 
Adv. J. Food Sci. Technol., 5(2): 127-131, 2013

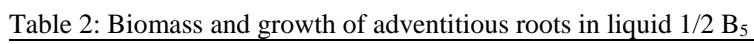

\begin{tabular}{lllll}
\hline Time of culture (days) & DW (g/L) & Growth ratio & $\begin{array}{l}\text { Biomass productivity between two } \\
\text { checked intervals (gDW/L/d) }\end{array}$ & $\begin{array}{l}\text { Final biomass } \\
\text { productivity (gDW/L/d) }\end{array}$ \\
\hline 0 & 1.120 & - & - & - \\
7 & 1.650 & 0.467 & 0.075 & - \\
15 & 5.680 & 2.442 & 0.504 & - \\
22 & 12.07 & 1.125 & 0.913 & - \\
30 & 13.45 & 0.114 & 0.173 & 0.411 \\
\hline This represents a typical result of three experiments & & &
\end{tabular}

Table 3: Comparison of total saponins content between different cultures and explants

\begin{tabular}{llll}
\hline Sample type & Content of total saponins $(\mathrm{mg} / \mathrm{g} \mathrm{DW})$ & Relative growth rate & $\begin{array}{l}\text { Yield of total saponins } \\
\text { (mg/L medium) }\end{array}$ \\
\hline Sterile seedlings & $17.85 \pm 1.25$ & $0.028 \pm 0.0079$ & 401.630 \\
Callus & $11.71 \pm 0.90$ & $0.034 \pm 0.0065$ & 263.750 \\
Adventitious roots & $16.33 \pm 0.85$ & $0.182 \pm 0.0082$ & 2041.25 \\
Liquid media & $0.540 \pm 0.10(\mathrm{mg} / \mathrm{mL})$ & - & 540.000 \\
\hline
\end{tabular}

This represents the mean value of three experiments plus S.D.

Table 4: Effects of elicitors on the growth and accumulation of adventitious roots of $P$. tunicoides

\begin{tabular}{lll}
\hline $\begin{array}{l}\text { Time of addition } \\
\text { of oxalate (day) }\end{array}$ & $\begin{array}{l}\text { Biomass of adventitious } \\
\text { roots }(\mathrm{g} / \mathrm{L} \mathrm{DW})\end{array}$ & $\begin{array}{l}\text { Contents of total } \\
\text { saponins }(\mathrm{mg} / \mathrm{L})\end{array}$ \\
\hline 0 & $8.12 \pm 0.250$ & $9.4 \pm 0.400$ \\
5 & $9.41 \pm 0.320$ & $10.8 \pm 0.62$ \\
10 & $12.50 \pm 0.58$ & $13.2 \pm 0.82$ \\
20 & $14.20 \pm 1.20$ & $15.6 \pm 1.54$ \\
\hline \multicolumn{2}{l}{ This represents the mean value of three experiments plus S.D. }
\end{tabular}

the medium has been extensively studied in plant cell cultures, there are only a few reports of elicitors being applied to hairy root cultures. Oxalic acid was a kind of secondary metabolites in plant cells and has been proven to be an efficient abiotic elicitor for the induction of tanshinone and depsides in Salvia miltiorrhiza suspension cells (Zhang et al., 2009), a series of pathogen resistance-related enzymes (Zhang et al., 1998a, b). Here, we first described the profile of the growth of adventitious roots affected by oxalic acid application in the mode of liquid culture. As shown in Table 4, it indicated clearly that the oxalic acid-caused effect on the growth rate of adventitious roots depends on the stage of growth at which they were treated. It retarded the growth of adventitious roots when oxalic acid was fed to the culture medium at the initial stage of culture, however, it could successfully stimulate a remarkable accumulation of total saponins without adverse effects on the growth of adventitious roots after addition of oxalate at the late stage of culture period (e.g., at the $20^{\text {th }}$ day of culture ). This result seems to be explained by the observations that the slight toxicity to the growth of plant cells caused by low concentration oxalate and its systematic induction on secondary metabolisms in whole seedlings (Zhang et al., 2009; Zhang et al., 1998a, b).

\section{ACKNOWLEDGMENT}

The authors thank the National Natural Science Foundation of China (No. 31070164), the MST Project of Innovation Fund for small and medium enterprises (No. 12C26214104239). We also thanks Dr. Wang Chao for his critical reading and correction of this manuscript, Prof. Fengxie Jin for his kind presentation of standard sample of $R e$.

\section{REFERENCES}

Cristofori, V., Y. Rouphael and E. Rugini, 2010. Collection time, cutting age, IBA and putrescine effects on root formation in Corylus avellana L. cuttings. Sci. Horticul., 124: 189-194.

Deng, X., X.X. Liu, D. Zhu and Q. Wang, 2009. A new triterpenoid saponin from Psammosilene tunicoides. Chinese J. Nat. Med., 7: 101-104.

Min, J.Y., H.Y. Jung and S.M. Kang, 2007. Production of tropane alkaloids by small-scale bubble column bioreactor cultures of Scopolia parviflora adventitious roots. Bioresour. Technol., 98: 1748-1753.

Staden, V.J. and A.R. Harty, 1988. Cytokinins and Adventitious Root Formation. In: Davis, T.D., B.E. Haissig and N. Sankhla (Eds.), Adventitious Root Formation in Cuttings. Dioscorides Press, Portland, pp: 185-201.

Sudha, C.G. and S. Seeni, 2001. Establishment and analysis of fast-growing normal root culture of Decalepis arayalpathra, a rare endemic medicinal plant. Curr. Sci., 81: 371-374.

Tian, P.W., T. Liu, F. Yang and Z.S. Zhang, 2009. Effects of oxalate on ginseng callus cells growth and the accumulation of ginseng polysaccharide and panax saponin. Lishizhen Med. Mater. Med. Res., 20: 2197-2199.

Wang, X., D. Qiu and C. Jiang, 2002. Advances in studies on the miao national herb Psammosilene tunicoides. Chinese J. Bas. Med. Tradit. Chinese Med., 8: 877-881.

Wang, X., Y. Zhang and J. Xu, 2006. Effects and mechanism of total saponins of Psammosilene tunicoides against rheumatoid arthritis. Chinese J. Chinese Mater. Med., 31: 419-421.

Wei, W., T.X. Liu and Z.S. Zhang, 2010. Effects of culture component on the callus growth and saponin accumulation in Psammosilenet unicoides. Lishizhen Med. Mater. Med. Res., 21: 1208-1210. 
Zhang, Z.S. and Z.Q. Wang, 2009. Effects of culture conditions on the saponin accumulation in Astragalus membranaceus callus. J. Dalian Polytechnic Univ., 28: 174-177.

Zhang, Z.S., X.X. Peng and Z.D. Jiang, 1998a. The systemic induction of peroxidase by oxalate in cucumber leaves. ACTA Phytopathol. Sinica, 28: $145-150$.

Zhang, Z.S., X.X. Peng and Z.D. Jiang, 1998b. Systemic induction of several pathogen-resistancerelated enzymes by oxalate in cucumber leaves. J. South China Agric. Univ., 19: 16-20.
Zhang, Z.S., P.W. Tian and T.X. Liu, 2009. Improvement of tanshinone and depsides accumulation in Salvia miltiorrhiza suspension culture cells by oxalate treatment. Chinese J. Chinese Mater. Med., 34(7): 919-921.

Zhong, J.J., Z.W. Pan, Z.Y. Wang, F. Chen, M. Takag and T. Yoshida, 2002. Effect of mixing time on taxoid production using suspension cultures of Taxus chinensis in a centrifugal impeller bioreactor. J. Biosci. Bioeng., 94: 244-250. 